\title{
Optimal Control Method for Solving the Cauchy-Neumann Problem for the Poisson Equation
}

\author{
H.F. Guliyev and Y.S. Gasimov \\ Institute of Applied Mathematics Baku State University \\ 23 Zahid Khalilov Str., Baku AZ1148, Azerbaijan \\ E-mail: hkuliyev@rambler.ru \\ ysfgasimov@yahoo.com \\ S.M. Zeynalli \\ Ganja State University \\ 187 Heydar Aliyev Ave., Ganja, AZ2000, Azerbaijan
}

Received September 27, 2013

\begin{abstract}
In the paper, the ill-posed Cauchy-Neumann problem is considered for the Poisson equation. The problem is reduced to the optimal control problem that is regularized. Optimization methods are applied to the solution of the obtained problem.

Key words: Ill-posed problem, Cauchy-Neumann problem, optimal control method, Poisson equation.

Mathematics Subject Classification 2010: 35J05, 35J25 (primary); 49J20, 49K20 (secondary).
\end{abstract}

\section{Introduction}

Optimal control methods have a wide field of applications such as modeling of various practical systems, studying of some classes of inverse and ill-posed problems $[2,3]$, etc. It is known that the Cauchy-Neumann problem for elliptic equations, particularly for the Poisson equation, is an ill-posed problem (see $[6$, 11]). The necessity to study the ill-posed problems of mathematical physics and their well-posed formulation was first noted by A.N. Tikhonov. It was stimulated by the fact that some physical processes mathematically are described by these problems. Systematic studying of these problems has begun since the 50-th of the last century, and various methods have been developed for their investigation.

In [1], optimal control methods are applied to the solution of the ill-posed Cauchy-Dirichlet problem for the Poisson equation. Here we consider the illposed Cauchy-Neumann problem for the Poisson equation that reduces to the

(c) H.F. Guliyev, Y.S. Gasimov, and S.M. Zeynalli, 2014 
solution of the optimal control problem with specially constructed functional. It takes us to the adjoint problem with naturally simple form.

Note that the interest to the studying of this problem is encouraged with the strong relations of the Poisson equation with several applications as well as problems of electrostatics, mathematical engineering, and theoretical physics.

\section{Problem Statement}

In the domain $Q=\{(x, t) \mid 0<x<\pi,-1<t<1\}$, consider the boundary problem

$$
\begin{gathered}
\frac{\partial^{2} u}{\partial x^{2}}+\frac{\partial^{2} u}{\partial t^{2}}=f(x, t), \quad(x, t) \in Q, \\
\left.\frac{\partial u(x, t)}{\partial x}\right|_{x=0}=0,\left.\frac{\partial u(x, t)}{\partial x}\right|_{x=\pi}=0, \quad t \in(-1,1), \\
\left.u(x, t)\right|_{t=-1}=\varphi_{0}(x),\left.\frac{\partial u(x, t)}{\partial t}\right|_{t=-1}=\varphi_{1}(x), x \in(0, \pi) .
\end{gathered}
$$

It is supposed that $\left.\frac{\partial u(x, t)}{\partial t}\right|_{t=1} \in U_{\partial}$, where $U_{\partial}$ is a convex closed set in $L_{2}(0, \pi)$, $0 \in U_{\partial}$ and $f \in L_{2}(Q), \varphi_{0} \in W_{2}^{1}(0, \pi), \varphi_{1} \in L_{2}(0, \pi)$ are given functions.

It is known that (2.1)-(2.3) is an ill-posed problem [4, 7].

Let us introduce an optimal control problem in correspondence with the problem above. For this purpose, we replace the conditions (2.3) by the following ones:

$$
\left.\frac{\partial u(x, t)}{\partial t}\right|_{t=-1}=\varphi_{1}(x),\left.\quad \frac{\partial u(x, t)}{\partial t}\right|_{t=1}=v(x), \quad x \in(0, \pi)
$$

and consider the problem on finding in $U_{\partial}$ the minimum of the functional

$$
J(v)=\int_{0}^{\pi}\left[u(x,-1)-\varphi_{0}(x)\right]^{2} d x
$$

subject to (2.1), (2.2), (2.4).

As is known from the general optimal control theory, (2.1), (2.2), (2.4), (2.5) is also an ill-posed problem. Note that if $f \in L_{2}(Q), \varphi_{1} \in L_{2}(0, \pi), v \in L_{2}(0, \pi)$, then the boundary problem $(2.1),(2.2),(2.4)$ has the unique solution from $W_{2}^{1}(Q)[5]$.

\section{Regularization of the Optimal Control Problem (2.1), (2.2), (2.4), (2.5)}

The regularization method is one of important techniques used for solving illposed problems $[5,10]$. We apply this method to solve the problem (2.1), (2.2), 
(2.4), (2.5). We take the functional $\varepsilon \int_{0}^{\pi}|v(x)|^{2} d x \quad(\varepsilon>0)$ as a stabilizer in the considering problem. Thus, for the minimization, we obtain the functional

$$
J_{\varepsilon}(v)=J(v)+\varepsilon \int_{0}^{\pi}|v(x)|^{2} d x=\int_{0}^{\pi}\left[u(x,-1)-\varphi_{0}(x)\right]^{2} d x+\varepsilon \int_{0}^{\pi}|v(x)|^{2} d x
$$

in the class $U_{\partial}$ subject to $(2.1),(2.2),(2.5)$.

Let $u(x, t ; v)$ be a solution of the problem $(2.1),(2.2),(2.4)$ corresponding to the given control $v \in U_{\partial} ; u(x, t ; 0)$ be a solution of the problem (2.1), (2.2), (2.4) for $v(x) \equiv 0$.

Let us define

$$
\begin{gathered}
a\left(v_{1}, v_{2}\right)=\int_{0}^{\pi}\left[u\left(x,-1 ; v_{1}\right)-u(x,-1 ; 0)\right]\left[u\left(x,-1 ; v_{2}\right)-u(x,-1 ; 0)\right] d x \\
+\varepsilon \int_{0}^{\pi} v_{1}(x) v_{2}(x) d x, \\
L(v)=\int_{0}^{\pi}\left[\varphi_{0}(x)-u(x,-1 ; 0)\right][u(x,-1 ; v)-u(x,-1 ; 0)] d x,
\end{gathered}
$$

where $a\left(v_{1}, v_{2}\right)$ is a bilinear continuous symmetric form on $U_{\partial} ; L(v)$ is a linear form on $U_{\partial}$.

Using these definitions and taking $v_{1}=v_{2}=v$ in the expression for $a\left(v_{1}, v_{2}\right)$, the functional (3.1) can be rewritten in the form

$$
J_{\varepsilon}(v)=a(v, v)-2 L(v)+\int_{0}^{\pi}\left[u(x,-1 ; 0)-\varphi_{0}(x)\right]^{2} d x
$$

Since $a\left(v_{1}, v_{2}\right)$ is a bilinear continuous symmetric form and it satisfies the condition (see definition for $a\left(v_{1}, v_{2}\right)$ )

$$
a(v, v) \geq \varepsilon\|v\|_{L_{2}}^{2},
$$

it follows from the well-known theorem from [8, page 13] that the theorem below is valid.

Theorem 3.1. For the optimal control problem (2.1), (2.2), (2.4), (3.1) there exists the element $\bar{v} \in U_{\partial}$ such that $J_{\varepsilon}(\bar{v})=\inf _{v \in U_{\partial}} J_{\varepsilon}(v)$ and this element is unique. 

below.

Basing on the theorem from [8, page 18$]$, we can easily prove the theorem

Theorem 3.2. For $\bar{v} \in U_{\partial}$ to be an optimal control, it is necessary and sufficient to fulfill the inequality

$$
J_{\varepsilon v}^{\prime}(\bar{v})(v-\bar{v}) \geq 0 \quad \forall v \in U_{\partial},
$$

which is equivalent to

$$
\begin{gathered}
\int_{0}^{\pi}\left[u(x,-1 ; \bar{v})-\varphi_{0}(x)\right] u_{v}(x,-1 ; \bar{v})[v(x)-\bar{v}(x)] d x \\
+\varepsilon \int_{0}^{\pi} \bar{v}(x)[v(x)-\bar{v}(x)] d x \geq 0 \quad \forall v \in U_{\partial},
\end{gathered}
$$

where $J_{\varepsilon v}^{\prime}$ is a Gateaux derivative with respect to $v ; u_{v}(x, t ; v)$ is a derivative of the solution of the problem (2.1), (2.2), (2.5) with respect to $v$.

Let us transform inequality (3.2). The linear boundary problem (2.1), (2.2), (2.4) can be rewritten in an operator form

$$
A u=F \equiv\left\{f, \varphi_{1}, v\right\},
$$

where $A$ is an unbounded linear operator from the space $L_{2}(Q)$ to the Hilbert space $L_{2}(Q) \times L_{2}(0, \pi) \times L_{2}(0, \pi)$,

$$
A: u(x, t) \mapsto\left\{\Delta u(x, t),\left.\frac{\partial u(x, t)}{\partial t}\right|_{t=-1},\left.\frac{\partial u(x, t)}{\partial t}\right|_{t=1}\right\} .
$$

As the domain of $A$, we take a set of functions from $W_{2}^{2}(Q)$ satisfying the conditions

$$
\left.\frac{\partial u(x, t)}{\partial x}\right|_{x=0}=0,\left.\quad \frac{\partial u(x, t)}{\partial x}\right|_{x=\pi}=0, \quad t \in(-1,1) .
$$

Then the operator $A$ admits the closure $\bar{A}$ that has an inverse. It means that the operator equation above has a generalized solution $u=\bar{A}^{-1} F$ belonging to $W_{2}^{1}(Q)[5,10]$.

Let us take a derivative of this solution in the direction $v-\bar{v}: u_{v}(x, t ; \bar{v})[v-\bar{v}]$ $=u(x, t ; v)-u(x, t ; \bar{v})$. 
Then inequality (3.2) takes the form

$$
\begin{gathered}
\int_{0}^{\pi}\left[u(x,-1 ; \bar{v})-\varphi_{0}(x)\right][u(x,-1 ; v)-u(x,-1 ; \bar{v})] d x \\
+\varepsilon \int_{0}^{\pi} \bar{v}(x)[v(x)-\bar{v}(x)] d x \geq 0 \quad \forall v \in U_{\partial} .
\end{gathered}
$$

\section{Optimality Condition}

Let us introduce the adjoint boundary problem

$$
\begin{gathered}
\frac{\partial^{2} \psi}{\partial x^{2}}+\frac{\partial^{2} \psi}{\partial t^{2}}=0, \quad(x, t) \in Q, \\
\left.\frac{\partial \psi(x, t)}{\partial x}\right|_{x=0}=\left.\frac{\partial u(x, t)}{\partial x}\right|_{x=\pi}=0, \quad t \in(-1,1), \\
\left.\frac{\partial \psi(x, t)}{\partial t}\right|_{t=-1}=u(x,-1 ; \bar{v})-\varphi_{0}(x),\left.\frac{\partial \psi(x, t)}{\partial t}\right|_{t=1}=0, x \in(0, \pi) .
\end{gathered}
$$

Note that the problem (4.1)-(4.3) has the unique solution from $W_{2}^{2}(Q)[5]$.

Using the boundary problem (4.1)-(4.3), we can transform the first term of inequality (3.3). If to take $\tilde{u}(x, t)=u(x, t ; v)-u(x, t ; \bar{v})$, then it is clear that

$$
\iint_{Q}\left[\frac{\partial^{2} \tilde{u}}{\partial x^{2}}+\frac{\partial^{2} \tilde{u}}{\partial t^{2}}\right] \psi(x, t ; \bar{v}) d x d t=0 .
$$

Integrating by parts in the above equation and taking into account (2.2), (2.4), (4.1)-(4.3), we have

$$
\begin{gathered}
\int_{Q} \int\left[\frac{\partial^{2} \tilde{u}}{\partial x^{2}}+\frac{\partial^{2} \tilde{u}}{\partial t^{2}}\right] \psi(x, t ; \bar{v}) d x d t=\int_{0}^{\pi}[v(x)-\bar{v}(x)] \psi(x, 1 ; \bar{v}) d x \\
+\left.\int_{0}^{\pi}[u(x,-1 ; v)-u(x,-1 ; \bar{v})] \frac{\partial \psi(x, t ; \bar{v})}{\partial t}\right|_{t=-1} d x=0,
\end{gathered}
$$

where $\psi(x, t ; \bar{v})$ is a solution of the problem (4.1)-(4.3) corresponding to the control $\bar{v} \in U_{\partial}$. 
Taking into account the first condition of (4.3) from (4.4), we can obtain

$$
\begin{gathered}
\int_{0}^{\pi}\left[u(x,-1 ; \bar{v})-\varphi_{0}(x)\right][u(x,-1 ; v)-u(x,-1 ; \bar{v})] d x \\
=-\int_{0}^{\pi} \psi(x, 1 ; \bar{v})[v(x)-\bar{v}(x)] d x .
\end{gathered}
$$

Then from (3.3) and (4.5) it follows that

$$
\int_{0}^{\pi}[-\psi(x, 1 ; \bar{v})+\varepsilon \bar{v}(x)][v(x)-\bar{v}(x)] d x \geq 0 \quad \forall v \in U_{\partial} .
$$

This proves the optimality condition in the form of the following theorem.

Theorem 4.1. Let the function $\bar{v}(x) \in U_{\partial}$ be an optimal control for the problem (2.1), (2.2), (2.4), (3.1). Then it is necessary and sufficient that this function satisfy the boundary problems (2.1), (2.2), (2.4), (4.1)-(4.3) and variational inequality (4.6).

\section{Application of the Fourier Method}

Now we analyze the boundary problems (2.1), (2.2), (2.4) and (4.1)-(4.3) by using the Fourier method. The applicability of this method to the considered problems is shown in [5]. We will look for the solutions of the boundary problems in the forms

$$
u(x, t)=u_{0}(t)+\sum_{k=1}^{\infty} u_{k}(t) X_{k}(x), \quad \psi(x, t)=\psi_{0}(t)+\sum_{k=1}^{\infty} \psi_{k}(t) X_{k}(x),
$$

where

$$
X_{0}(x)=\frac{1}{\sqrt{\pi}}, \lambda_{o}=0, X_{k}(x)=\sqrt{\frac{2}{\pi}} \cos k x ; \lambda_{k}=-k^{2}, \quad k=1,2, \ldots
$$

are the systems of orthonormal eigenfunctions and eigenvalues of the spectral problem

$$
X^{\prime \prime}(x)=\lambda X(x), X^{\prime}(0)=X^{\prime}(\pi)=0 .
$$

From (2.1), (2.2), (2.4), (4.1)-(4.3) and (4.6) we obtain

$$
\left\{\begin{array}{l}
\ddot{u}_{k}(t)-k^{2} u_{k}(t)=f_{k}(t), \quad t \in(-1,1) \\
\dot{u}_{k}(-1)=\varphi_{1 k}, \quad \dot{u}_{k}(1)=\bar{v}_{k}, \quad k=0,1,2, \ldots
\end{array}\right.
$$




$$
\begin{aligned}
& \left\{\begin{array}{l}
\ddot{\psi}_{k}(t)-k^{2} \psi_{k}(t)=0, \quad t \in(-1,1), \\
\dot{\psi}_{k}(-1)=u_{k}(-1)-\varphi_{0 k}, \dot{\psi}_{k}(1)=0, \quad k=0,1,2, \ldots,
\end{array}\right. \\
& {\left[-\psi_{k}(1)+\varepsilon \bar{v}_{k}\right]\left[v_{k}-\bar{v}_{k}\right] \geq 0 \quad \forall v_{k}, \quad k=0,1,2, \ldots,}
\end{aligned}
$$

where $f_{k}(t), \varphi_{0 k}, \varphi_{1 k}, \bar{v}_{k}, v_{k}, k=0,1,2, \ldots$ are Fourier coefficients of the functions $f(x, t), \varphi_{0}(x), \varphi_{1}(x), \bar{v}(x), v(x)$ with respect to the system (5.1).

From the general theory of the boundary problems for the ordinary differential equations [9], we can conclude that the solutions of the boundary problem (5.2) for $k=1,2, \ldots$ can be written in the form

$$
u_{k}(t)=\bar{v}_{k} \frac{\operatorname{ch} k(1+t)}{k \operatorname{sh} 2 k}-\varphi_{1 k} \frac{\operatorname{ch} k(1-t)}{k \operatorname{sh} 2 k}+\int_{-1}^{1} G_{k}(t ; \tau) f_{k}(\tau) d \tau,
$$

where

$$
G_{k}(t, \tau)=\left\{\begin{array}{l}
-\frac{\operatorname{ch} k(1-\tau) \operatorname{ch} k(1+t)}{k \operatorname{sh} 2 k}, t \in[-1, \tau] \\
\frac{1}{k} \operatorname{sh} k(t-\tau)-\frac{\operatorname{ch} k(1-\tau) \operatorname{ch} k(1+t)}{k \operatorname{sh} 2 k}, t \in[\tau, 1]
\end{array}\right.
$$

is a Green function for the problem (5.2).

For the problem (5.3), we have

$$
\psi_{k}(t)=-\frac{u_{k}(-1)-\varphi_{0 k}}{k \operatorname{sh} 2 k} \operatorname{ch} k(1-t) .
$$

For $k=0$, the solutions of the problems (5.2), (5.3) are in the forms

$$
\begin{gathered}
u_{0}(t)=\int_{-1}^{t}(t-s) f_{0}(s) d s+\varphi_{10}(t+1)+\varphi_{00}, \\
\psi_{0}(t)=c,
\end{gathered}
$$

where $c$ is a random constant and $\int_{-1}^{1} f_{0}(t) d t+\varphi_{10}=\bar{v}_{0}$, moreover, $\varphi_{00}=\frac{1}{\sqrt{\pi}} \int_{0}^{\pi} \varphi_{0}(x) d x, \varphi_{10}=\frac{1}{\sqrt{\pi}} \int_{0}^{\pi} \varphi_{1}(x) d x, \quad \bar{v}_{0}=\frac{1}{\sqrt{\pi}} \int_{0}^{\pi} v(x) d x, \quad f_{0}(t)=$ $\frac{1}{\sqrt{\pi}} \int_{0}^{\pi} f(x, t) d x$.

From (5.5), (5.6) and (5.4), we get

$$
\begin{gathered}
u_{k}(-1)=\frac{\bar{v}_{k}}{k \operatorname{sh} 2 k}-\varphi_{1 k} \frac{\operatorname{cth} 2 k}{k}+\int_{-1}^{1} G_{k}(-1 ; \tau) f_{k}(\tau) d \tau, \quad k=1,2, \ldots, \\
-\psi_{k}(1)=\frac{u_{k}(-1)-\varphi_{0 k}}{k \operatorname{sh} 2 k}, \quad k=1,2, \ldots,
\end{gathered}
$$




$$
\left[u_{k}(-1)-\varphi_{0 k}+\varepsilon k \operatorname{sh} 2 k \cdot \bar{v}_{k}\right]\left[v_{k}-\bar{v}_{k}\right] \geq 0 \forall v_{k}, \quad k=1,2, \ldots .
$$

The condition (5.7) can be transformed as

$$
\begin{aligned}
& {\left[-\varphi_{1 k} \frac{\operatorname{cth} 2 k}{k}+\bar{v}_{k}\left(\frac{1}{k \operatorname{sh} 2 k}+\varepsilon k \operatorname{sh} 2 k\right)-\varphi_{o k}+\int_{-1}^{1} G_{k}(-1 ; \tau) f_{k}(\tau) d \tau\right]} \\
& \times\left(v_{k}-\bar{v}_{k}\right) \geq 0 \forall v_{k}, \quad k=1,2, \ldots .
\end{aligned}
$$

Now let us consider the case $U_{\partial}=L_{2}(0, \pi)$. Then from $\left(5.7^{\prime}\right)$ we obtain

$$
\bar{v}_{k}=\beta_{k \varepsilon}^{-1}\left[\varphi_{0 k}+\varphi_{1 k} \frac{\operatorname{cth} 2 k}{k}-\int_{-1}^{1} G_{k}(-1 ; \tau) f_{k}(\tau) d \tau\right],
$$

where

$$
\beta_{k \varepsilon}=\frac{1+\varepsilon k^{2} \operatorname{sh}^{2} 2 k}{k \operatorname{sh} 2 k}, \quad k=1,2, \ldots .
$$

As follows from (5.4), $\bar{v}_{0}=\frac{\psi_{0}(1)}{\varepsilon}$ for $k=0$.

Since we are interested in the bounded value of $\bar{v}_{0}$ for $\varepsilon \rightarrow 0$, the value $\psi_{0}(1)$ should be equal to zero. Then $\bar{v}_{0}=0$, and the solution of the problem (5.3) for $k=0$ is equal to zero.

Since the solution of the problem (5.2) for $k=0$ has the form

$$
u_{0}(t)=\int_{-1}^{t}(t-s) f_{0}(s) d s+\varphi_{10}(t+1)+\varphi_{00},
$$

it is necessary to fulfill the condition $\int_{-1}^{1} f_{0}(t) d t+\varphi_{10}=0$.

Thus, we have the optimal values of the Fourier coefficients $\bar{v}_{k}$ for the function $\bar{v}(x)$. Then, for $\varepsilon \rightarrow 0$ from (5.5), (5.8), we have

$$
\begin{gathered}
u_{k 0}(t)=\lim _{\varepsilon \rightarrow 0} u_{k}(t)=\varphi_{0 k} \operatorname{ch} k(1+t)+\frac{\varphi_{1 k}}{k \operatorname{sh} 2 k}[\operatorname{ch} 2 k \operatorname{ch} k(1+t)-\operatorname{ch} k(1-t)] \\
-\operatorname{ch} k(1+t) \int_{-1}^{1} G_{k}(-1 ; \tau) f_{k}(\tau) d \tau+\int_{-1}^{1} G_{k}(t ; \tau) f_{k}(\tau) d \tau, k=1,2, \ldots \\
\bar{v}_{k 0}=\lim _{\varepsilon \rightarrow 0} \bar{v}_{k}=\varphi_{0 k} k \operatorname{sh} 2 k+\varphi_{1 k} \operatorname{ch} 2 k-k \operatorname{sh} 2 k \int_{-1}^{1} G_{k}(-1 ; \tau) f_{k}(\tau) d \tau, k=1,2, \ldots
\end{gathered}
$$


Note that the solution $u_{k}(t)$ given by (5.5) and corresponding to the optimal Fourier coefficients $\bar{v}_{k}, k=1,2, \ldots$ given by (5.8), satisfies the limit relations $\lim _{\varepsilon \rightarrow 0} u_{k}(-1)=\varphi_{0 k}$. This is consistent with the condition $u(x,-1)=\varphi_{0}(x)$ from $(2.3)$.

Thus the exact solution of the problem (2.1), (2.2), (2.4), (2.5) has the form

$$
\bar{v}(x)=\sum_{k=1}^{\infty} \sqrt{\frac{2}{\pi}} \operatorname{sh} 2 k\left[k \varphi_{0 k}+\varphi_{1 k} \operatorname{cth} 2 k-k \int_{-1}^{1} G_{k}(-1 ; \tau) f_{k}(\tau) d \tau\right] \cos k x .
$$

Then the solution of the initial problem (2.1)-(2.3) has the form

$$
\begin{gathered}
u(x, t)=\frac{1}{\sqrt{\pi}} u_{0}(t) \\
+\sum_{k=1}^{\infty} \sqrt{\frac{2}{\pi}}\left\{\varphi_{0 k} \operatorname{ch} k(1+t)+\frac{\varphi_{1 k}}{k \operatorname{sh} 2 k}[\operatorname{ch} 2 k \operatorname{ch} k(1+t)-\operatorname{ch} k(1-t)]\right. \\
\left.-\operatorname{ch} k(1+t) \int_{-1}^{1} G_{k}(-1 ; \tau) f_{k}(\tau) d \tau+\int_{-1}^{1} G_{k}(t ; \tau) f_{k}(\tau) d \tau\right\} \cos k x
\end{gathered}
$$

Now let us consider the analogue of the Hadamard example for the problem (2.1)-(2.3). For this purpose we take

$$
f(x, t)=0, \varphi_{0}(x)=0, \varphi_{1}(x)=\frac{1}{k} \exp \{-\sqrt{k}\} \cos k x, \quad k \in N .
$$

In this case, the solution of the Cauchy-Neumann problem for the Laplace equation is unique and has the form

$$
u(x, t)=\frac{1}{k^{2}} \exp \{-\sqrt{k}\} \cos k x \operatorname{sh} k(1+t), \quad k \in N .
$$

At the same time, for $k \rightarrow \infty$, the function $\varphi_{1}(x)$ tents to zero uniformly and all its derivatives belong to $L_{2}(0, \pi)$. However, the solution (5.11) for any $t>-1$ has a cosinusodial form with arbitrary large amplitude not belonging to $L_{2}(Q)$.

For the function $\varphi_{1}(x)$ to satisfy $\left\{\exp \{2 k\} \varphi_{1 k}\right\}_{k=1}^{\infty} \in l_{2}$, it is necessary and sufficient that the Fourier coefficients $\varphi_{1 k}$ have asymptotics of order $\exp \{-(2+\varepsilon) k\}$ for sufficiently large $k$, where $\varepsilon>0$. In our case, we have only asymptotics of order $\exp \{-\sqrt{k}\}$ that is not enough to provide the well-posedness of the Cauchy-Neumann problem for the Laplace equation. 


\section{References}

[1] M.M. Amangaliyeva, M.T. Janaliyev, and K.B. Imanberdiyev, On an Optimization Method to the Solution of the Cauchy-Dirichlet Problem for the Poisson Equation. - J. Inv. Ill-Posed Problems 15 (2007), 1-10.

[2] Y.S. Gasimov, On a Shape Design Problem for One Spectral Functional. - J. Inv. Ill-Posed Problems 21 (2013), No. 5, 629-637.

[3] R.S. Gurbanov, N.B. Nuriyev, and R.S. Gurbanov, Technological Control and Optimization Problems in Oil Production: Theory and Practice. - Appl. Comput. Math. 12 (2013), No. 3, 314-324.

[4] S.I. Kabanikhin, Inverse and ill-Posed Problems. Syb. Sci. Publishing, Novosibirsk, 2009.

[5] O.A. Ladijenskaya, Boundary Problems of the Mathematical Physics. Nauka, Moscow, 1973.

[6] M.M. Lavrentev, On a Cauchy Problem for the Poisson Equation. - Izv. AS USSR, ser. Math. 20 (1955), No. 6, 819-842.

[7] R. Lattes and J-L. Lions, Quazi-Reversibility Method and its Applications. Mir, Moscow, 1970.

[8] J.L. Lions, Optimal Control of the Systems Described by the Partial Differential Equations. Nauka, Moscow, 1972.

[9] M.A. Naimark, Linear Differential Operators. Nauka, Moscow, 1969.

[10] V.A. Trenogin, Functional Analiysis. Nauka, Moscow, 1980.

[11] A.N. Tikhonov and V.Ya.R. Arsenin, Methods to the Solution of the ill-Posed Problems. Nauka, Moscow, 1974.

[12] F.P. Vasilev, Methods of Optimization. Faktorial, Moscow, 2002. 